\title{
CONCERNING REAL NUMBERS WHOSE POWERS HAVE NONINTEGRAL DIFFERENCES
}

\author{
HERMAN J. COHEN AND FRED SUPNICK
}

Various classes of real numbers $\alpha$ have been considered for which $\alpha^{s}-\alpha^{r}(s, r$ positive integers, $s \neq r$ ) is never integral (see pp. 244-246 of [1]). This note adds another class to this category.

If we consider the numbers $\alpha=a+b(2)^{1 / 2}$, we find that if $a$ and $b$ are positive and rational, $a \geqq 1$, then $\alpha^{s}-\alpha^{r}$ is nonintegral. This result is now generalized:

THEOREM. Let $\beta$ be any algebraic number having a minimal polynomial of the form:

$$
x^{n}-b_{1} x^{n-1}-b_{2} x^{n-2}-\cdots-b_{n-1} x-b_{n},
$$

where each $b_{i}$ is rational, $b_{i} \geqq 0, n>1$. Let $\alpha$ be defined by

$$
\alpha=c_{0}+c_{1} \beta^{d_{1}}+c_{2} \beta^{d_{2}}+\cdots+c_{m} \beta^{d_{m}},
$$

where each $c_{i}>0, c_{0} \geqq 1, m \geqq 1,1 \leqq d_{1}<d_{2}<\cdots<d_{m}<n ; c_{i}$ rational, $d_{i}$ integral.

Then $\alpha^{s}-\alpha^{r}$ is nonintegral for all positive integers $s, r(s \neq r)$.

Proof. Let us suppose that

$$
\alpha^{8}-\alpha^{r}-k=0,
$$

with $s>r$ and $k$ integral. Now,

$$
\alpha^{s}=\sum_{\Sigma_{0}^{m} q_{i=8}} \frac{s !}{q_{0} ! q_{1} ! \cdots q_{m} !}\left(c_{0}\right)^{q_{0}}\left(c_{1} \beta^{d_{1}}\right)^{q_{1}}\left(c_{2} \beta^{d_{2}}\right)^{q_{2}} \cdots\left(c_{m} \beta^{d_{m}}\right)^{q_{m}},
$$

while

$$
\alpha^{r}=\sum_{\Sigma_{0}^{m} t_{i}=r} \frac{r !}{t_{0} ! t_{1} ! \cdots t_{m} !}\left(c_{0}\right)^{t_{0}}\left(c_{1} \beta^{d_{1}}\right)^{t_{1}}\left(c_{2} \beta^{d_{2}}\right)^{t_{2}} \cdots\left(c_{m} \beta^{d_{m}}\right)^{t_{m}} .
$$

The right member of (5) contains the term for which $t_{0}=r-1$, $t_{1}=1$, and all other $t_{i}=0$, namely,

$$
r\left(c_{0}\right)^{r-1} c_{1} \beta^{d_{1}}
$$

similarly, the right member of (4) contains the term

$$
s\left(c_{0}\right)^{s-1} c_{1} \beta^{d_{1}} .
$$

Presented to the Society, August 29, 1962; received by the editors June 1, 1962. 
We note that the coefficient of $\beta^{d_{1}}$ in (7) exceeds that in (6).

In the same way, suppose we select any particular term

$$
\frac{r !}{t_{0} ! t_{1} ! \cdots t_{m} !}\left(c_{0}\right)^{t_{0}}\left(c_{1} \beta^{d_{1}}\right)^{t_{1}}\left(c_{2} \beta^{d_{2}}\right)^{t_{2}} \cdots\left(c_{m} \beta^{d_{m}}\right)^{t_{m}}
$$

in the right member of $(5)$, where $\sum_{0}^{m} t_{i}=r$. Now, let us associate with (8) the term

$$
\frac{s !}{q_{0} ! q_{1} ! \cdots q_{m} !}\left(c_{0}\right)^{q_{0}}\left(c_{1} \beta^{d_{1}}\right)^{q_{1}}\left(c_{2} \beta^{d_{2}}\right)^{q_{2}} \cdots\left(c_{m} \beta^{d_{m}}\right)^{q_{m}}
$$

of (4), where we pick $q_{0}=t_{0}+s-r, q_{i}=t_{i}$ for $1 \leqq i \leqq m$, so that $\sum_{0}^{m} q_{i}=s$.

We note that (8) and (9) both contain the same total exponent of $\beta$, namely $\sum_{1}^{m} d_{i} t_{i}$, but (except for the case $c_{0}=1, t_{1}=t_{2}=\cdots$. $\left.=t_{m}=0\right)$ the coefficient in (9) is greater than that in (8). Also, any two terms of (5) have distinct corresponding terms in (4). Consequently, substituting (4) and (5) into (3), we obtain an equation in $\beta$ of the form

$$
g_{0} \beta^{d_{m} s}+g_{1} \beta^{d_{m s-1}}+\cdots+g_{d_{m} s-d_{1}} \beta^{d_{1}}+\cdots+g_{d_{m^{s-1}} \beta}+g_{d_{m^{s}}}=0
$$

where $g_{i} \geqq 0$ for $i=0,1,2, \cdots, d_{m} s-1$, and $g_{d_{m} s-d_{1}}>0$.

Now, by (1), each power of $\beta$ in (10) can be expressed in terms of the basis $\left\{1, \beta, \beta^{2}, \cdots, \beta^{n-1}\right\}$ with nonnegative coefficients. After these substitutions have been made, the total coefficient of $\beta^{d_{1}}$ will still be positive. Thus (10) becomes a polynomial in $\beta$ whose degree $d$ satisfies $1 \leqq d_{1} \leqq d<n$, which is impossible.

\section{REFERENCE}

1. F. Supnick, H. J. Cohen and J. F. Keston, On the powers of a real number reduced modulo one, Trans. Amer. Math. Soc. 94 (1960), 244-257.

City College 